\title{
New 5-adic Cantor sets and fractal string
}

\author{
Ashish Kumar $^{1 *}$, Mamta Rani $^{2}$ and Renu Chugh ${ }^{1}$
}

\begin{abstract}
In the year (1879-1884), George Cantor coined few problems and consequences in the field of set theory. One of them was the Cantor ternary set as a classical example of fractals. In this paper, 5-adic Cantor one-fifth set as an example of fractal string have been introduced. Moreover, the applications of 5-adic Cantor one-fifth set in string theory have also been studied.
\end{abstract}

Keywords: Cantor one-fifth set; p-adic integers; 5-adic Cantor one-fifth set; Iterated function system (IFS); Fractal string

\section{Introduction}

During the late eighteenth century, mathematicians delighted in producing sets with ever more weird properties, many of them now recognized to be fractal in nature (Crilly et al.). George Cantor (1879-1884) wrote a series of papers entitled "Uber unendliche lineare punktmannichfaltigkeiten" (Cantor 1879; 1880; 1882; 1883a; 1883b; 1884) that contained the first systematic treatment of the point set topology of real line, in which he triggered some problems and consequences in the field of set theory. One of these is the classical Cantor set problem devised by Cantor in the footnote to a statement saying that perfect sets do not need to be everywhere dense (Fleron 1994). In last two decades, Devil's and other researchers established the graphical representation of Cantor sets in the form of staircases (Horiguchi and Morita 1984a; 1984b; Rani and Prasad 2010).

Middle one-third, a classical Cantor set found a celebrated place in the mathematical analysis and in its applications (Hutchinson 1981; Mendes 1999; Shaver 2010). For a fundamental work on Cantor set and its applications, one may refer to (Peitgen et al. 2004), (Devaney 1992), (Beardon 1965), (Falconer 1985), (Lapidus and van Frankenhuijsen 2006), (Gutfraind et al. 1990) and (Lee 1998). In recent years, $\mathrm{p}$-adic analysis has been used in various areas of mathematics as well as in aspects of quantum physics and string theory (Lapidus and van Frankenhuijsen 2006). For a detailed analysis of fractal string and p-adic integers, one may refer to (Chistyakov 1996; Hung 2007; Koblitz 1984; Robert 2000; Schikhof 1984; Vladimirov et al. 1994).

\footnotetext{
* Correspondence: akrmsc@gmail.com

'Department of Mathematics, Maharshi Dayanand University, Rohtak 124001, Haryana, India

Full list of author information is available at the end of the article
}

Lapidus and van Frankenhuijsen (2000; 2006) introduced the concept of fractal string and established the geometric zeta function, zeros of zeta function, spectra of fractal string and the complex dimension of the fractal string. In 2008, (Lapidus 2008) suggested that fractal string and their quantization may be related to aspects of string theory. In last few decades, M. L. Lapidus, jointly with other researchers generalized and introduced the various properties of fractal string (see (Edgar 2008; Lapidus 1992; Lapidus and Maier 1995; Lapidus and Pearse 2006; 2008; Lapidus and Pomerance 1993)).

In 2008, (Lapidus and Hung 2008; 2009) provided a framework for unifying the archimedean and p-adic (nonarchimedean) fractal string with their geometric zeta functions and complex dimensions for 3-adic Cantor sets and also the general case for p-adic Cantor sets respectively. Recently, (Ashish, Mamta Rani and Renu Chugh, Variants of Cantor Sets Using IFS, submitted and Ashish, Mamta Rani and Renu Chugh, Study of Variants of Cantor sets., submitted) studied the variants of Cantor sets and established their mathematical analysis using mathematical feedback system and iterated function system respectively.

Our goal in this paper is to study the Cantor one-fifth set as a new classical example of fractal string. Moreover, the non-archimedean (5-adic) Cantor one-fifth set with their applications in string theory has also been established. In the third section, the main results of our study have been presented, followed by the "Concluding remarks" section.

\section{Preliminaries}

In this section, we recall some basic definitions pertaining to the notion of (ordinary) fractal string and introduce

\section{Springer}


several new ones such as the most important of which are quinary expansion and Cantor one-fifth set:

\section{Definition 2.1. Cantor one-fifth set}

The Cantor one-fifth set for unequal intervals is defined as the $F=\cap F_{n+1}$, where $F_{n+1}$ is constructed by dividing $F_{n}$ in five unequal line segments and removing second and fourth one-fifth line segment, $F_{0}$ being the closed interval $0 \leq x \leq 1$ (Ashish, Mamta Rani and Renu Chugh, Variants of Cantor Sets Using IFS, submitted).

\section{Definition 2.2. Quinary expansion}

The sequence $0 x_{1} x_{2} x_{3} x_{4} x_{5} \ldots$, where each $x_{i}$ is either 0 , $1,2,3$, or 4 is called quinary expansion of $x$ if $x=x_{1} / 5+$ $x_{2} / 5^{2}+x_{3} / 5^{3}+\ldots$.

For example, the sequence $0.04444 \ldots$ is the quinary expansion of $1 / 5$ since we have

$$
\frac{0}{5}+\frac{4}{5^{2}}+\frac{4}{5^{3}}+\frac{4}{5^{4}}+\frac{4}{5^{5}}+\frac{4}{5^{6}}+\ldots \ldots=\frac{4}{5^{2}} \sum_{i=0}^{\infty} \frac{1}{5^{i}}=\frac{1}{5}
$$

Lapidus and van Frankenhuijsen (2000) and (2006), introduced the concept of fractal strings as follows:

\section{Definition 2.3. Fractal string}

A fractal string $\Omega$ is a bounded open subset of the real line $R$. The collection of lengths $\ell_{j}$ of the disjoint intervals is denoted by $L$.

For example, the complement of the Cantor set in the closed unit interval $[0,1]$ is a Cantor string. Moreover, the topological boundary of Cantor string is the Cantor set $C$ itself.

\section{Definition 2.4. Geometric zeta function}

The geometric zeta function of a fractal string $\Omega$ with lengths $L$ is

$$
\varsigma_{L}(s)=\sum_{k=1}^{\infty} m_{k} \ell_{k}^{s}
$$

where $\ell_{1}, \ell_{2}, \ldots, \ell_{k}$ are the lengths of open intervals and $m_{k}$ be the corresponding multiplicity of open intervals (Lapidus and van Frankenhuijsen 2000).

For example, Cantor string consists of intervals of lengths $\ell_{1}=\left(l_{1}=1 / 3\right), \ell_{2}=\left(l_{2}=l_{3}=1 / 9\right), \ell_{3}=\left(l_{4}=l_{5}=l_{6}=l_{7}=1 / 27\right)$, and so on, that is, the lengths are the numbers $3^{-k-1}$ with multiplicity $m_{3^{-k-1}}=2^{k}$ for $k=0,1,2,3, \ldots$. . So, the geometric zeta function is:

$$
\varsigma_{L}(s)=\sum_{k=0}^{\infty} m_{k} \ell_{k}^{s}=\sum_{k=0}^{\infty} 2^{k} \cdot 3^{(-k-1) s}=\frac{3^{-s}}{1-2.3^{-s}} \quad \text { for } \operatorname{Re}(s>D)
$$

where $\mathrm{D}=\log 2 / \log 3$ is the dimension of usual Cantor set.

Recently, (Ashish, Mamta Rani and Renu Chugh, Variants of Cantor Sets Using IFS, submitted), established the self-similarity of the Cantor one-fifth set using the iteration function system as follows:

\section{Theorem 2.1}

Let $f_{1}, f_{2}$ and $f_{3}$ be the similarity contraction mappings on $\mathbb{R}$ defined by

$$
f_{1}(x)=x / 5, \quad f_{2}(x)=(x+2) / 5, \quad f_{3}(x)=(x+4) / 5,
$$

where all the mappings have the ratio $1 / 5$. Then, the Cantor one-fifth set $F$ satisfies the self-referential equation

$$
F=f_{1}[F] \cup f_{2}[F] \cup f_{3}[F]
$$

for the iterated function system $\left(f_{1}, f_{2}, f_{3}\right)$.

\section{Main results}

5-adic (nonarchimedean) Cantor one-fifth set

A sequence $\left(s_{i}\right)_{i \in N}$ of natural numbers between 0 and $p$ 1 (inclusive) is a $p$-adic integer. We write this conventionally as ................s $s_{2} s_{1} s_{0}$. If ' $n$ ' is any natural number, and

$$
n=\overline{s_{k-1} s_{k-2} \ldots s_{1} s_{0}}
$$

is its $p$-adic representation (in other words, $n=\sum_{i=0}^{k-1} s_{i} p^{i}$ with each $s_{i}$ is a $p$-adic digit), then we identify ' $n$ ' with the $p$-adic integer $\left(s_{i}\right)$ with $s_{i}=0$ if $i \geq k$ (Madore 2000). Further, the set of $p$-adic integers, which we call $Z_{p}$ with two binary operations on it (addition and multiplication) is a ring. The relation between the set (ring) $Z_{p}$ of $p$-adic integers and the set (field) $\mathbb{Q}_{p}$ of $p$-adic numbers is the same as between the set (ring) $Z$ of integers and the set (field) $\mathbb{Q}$ of rationals (Madore 2000). Since, $Z_{p}$ is an important subspace of $\mathbb{Q}_{p}$, it can be represented as follows:

$Z_{p}=\left\{s_{0}+s_{1} p^{1}+s_{2} p^{2}+\ldots ; s_{i} \in(0,1,2, \ldots, p-1)\right.$, for all $\left.i \geq 0\right\}$

For this $p$-adic expansion, we can also write

$$
Z_{p}=\bigcup_{c=0}^{p-1}\left(c+p Z_{p}\right)
$$

where $c+p Z_{p}=\left\{y \in \mathbb{Q}_{p}:|y-c|_{p} \leq 1 / p\right\}$ (Lapidus and van Frankenhuijsen 2006) It is also known that there are topological models of $\mathbb{Z}_{p}$ in the Euclidean space $\mathbb{R}^{d}$ as fractal spaces such as the Cantor set and the Sierpinsky gasket (Robert 2000), where $\mathbb{Z}_{p}$ is homeomorphic to the ternary Cantor set. Now, we consider the ring of 5 -adic integers $Z_{5}$, that is, homeomorphic to Cantor one-fifth set.

Figure 1 below shows the representation of 5 -adic Cantor one-fifth set ' $N$. To start the construction, initiator $N_{0}=Z_{5}$ is subdivided into five equal subintervals $0+5 Z_{5}, 1+$ $5 Z_{5}, 2+5 Z_{5}, 3+5 Z_{5}$ and $4+5 Z_{5}$. Drop the subintervals $1+5 Z_{5}$ and $3+5 Z_{5}$ and repeat the same process for the remaining subintervals. Further, repeating the same process 


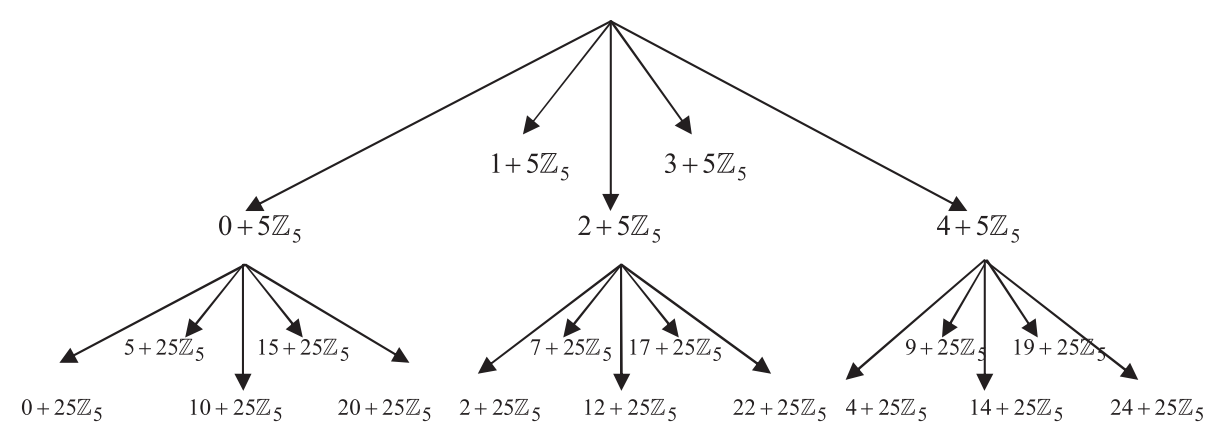

Figure 1 5-adic (nonarchimedean) Cantor one-fifth set.

over and over again, by removing the open subintervals of second and fourth position at each step from each closed interval, we obtain a sequence $N_{k}$ for $k=1,2, \ldots$ The 5adic Cantor one-fifth set (see Figure 1) $N_{k}$ consists of $3^{k}$ disjoint closed intervals. Thus, the 5-adic Cantor one-fifth set would be the limit ' $N$ ' of the sequence $N_{k}$ of sets. So, we define limit ' $N$ ' as the intersection of the sets $N_{k}$ i.e.

$$
N=\bigcap_{k \in N} N_{k}
$$

\section{Theorem 3.1}

Let $f_{1}, f_{2}$ and $f_{3}$ be the similarity contraction mappings on 5-adic integer $\mathbb{Z}_{5}$ defined by

$$
f_{1}(x)=5 x, \quad f_{2}(x)=5 x+2, \quad f_{3}(x)=5 x+4,
$$

with scaling ratio $1 / 5$. Then, the 5 -adic Cantor one-fifth set $N$ satisfies the self-referential equation

$$
N=f_{1}[N] \cup f_{2}[N] \cup f_{3}[N] .
$$

Proof: Using above construction of 5-adic Cantor onefifth set, we can say that

$$
N_{k+1}=f_{1}\left[N_{k}\right] \cup f_{2}\left[N_{k}\right] \cup f_{3}\left[N_{k}\right]
$$

for all $k \geq 1$. Since, the mapping $f_{j}$ for $j=1,2,3$ is oneto-one and $N=\cap N_{k}$, then it implies that $f_{j}[N]=f_{j}\left[\cap N_{k}\right]=\cap f_{j}\left[N_{k}\right]$, for $k=1,2, \ldots$

so that, we can write $f_{1}[N]=\cap f_{1}\left[N_{k}\right], f_{2}[N]=\cap f_{2}\left[N_{k}\right]$ and $f_{3}[N]=\cap f_{3}\left[N_{k}\right]$,

therefore, $f_{1}[N] \cup f_{2}[N] \cup f_{3}[N]=\left(\cap f_{1}\left[N_{k}\right]\right) \cup\left(\cap f_{2}\left[N_{k}\right]\right) \cup$ $\left(\cap f_{3}\left[N_{k}\right]\right)$

$$
\begin{aligned}
& f_{1}[N] \cup f_{2}[N] \cup f_{3}[N]=\cap\left(f_{1}\left[N_{k}\right] \cup f_{2}\left[N_{k}\right] \cup f_{3}\left[N_{k}\right]\right) \\
& f_{1}[N] \cup f_{2}[N] \cup f_{3}[N]=\cap N_{k+1}=N \\
& f_{1}[N] \cup f_{2}[N] \cup f_{3}[N]=N
\end{aligned}
$$

which gives the proof of the theorem.

Figure 2 shows the graphical representation of 5 -adic Cantor one-fifth set using iterated function system $\left(f_{1}\right.$, $\left.f_{2}, f_{3}\right)$.

\section{Quinary expansion of 5-adic Cantor one-fifth set Theorem 3.2}

The 5-adic Cantor one-fifth set is represented by the quinary expansion of its elements in the form

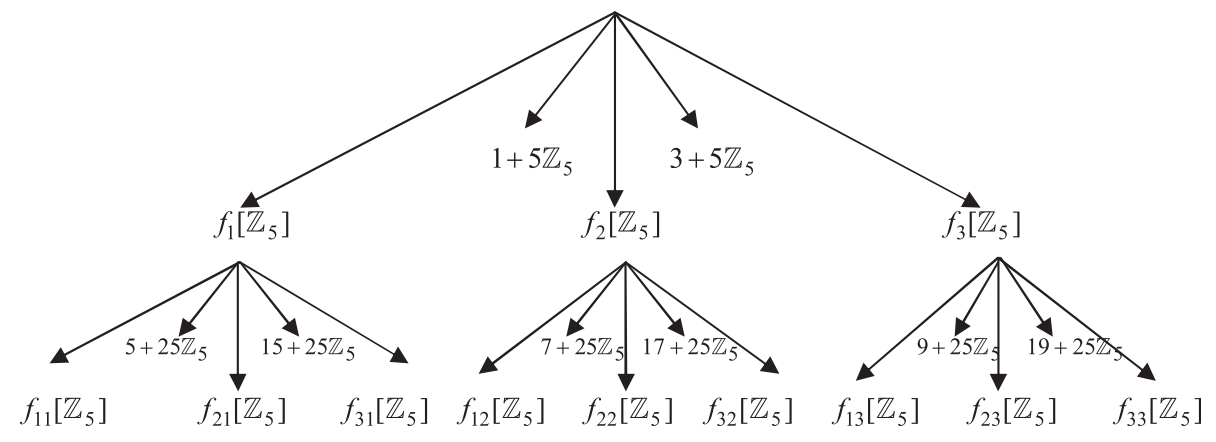

Figure 2 5-adic Cantor one-fifth set using IFS. 


$$
N=\left\{x \in \mathbb{Z}_{5}: x=x_{0}+5^{1} x_{1}+5^{2} x_{2}+\ldots, x_{j} \in\{0,2,4\}\right\}
$$

for all $j=0,1,2, \ldots .$.

Proof: Let us define the inverse of similarity contraction mappings $f_{1}, f_{2}$ and $f_{3}$, on $Z_{5}$ as follows:

$$
f_{1}^{-1}(x)=x / 5, \quad f_{2}^{-1}(x)=(x-2) / 5, \quad f_{3}^{-1}(x)=(x-4) / 5,
$$

Now, for $x_{j} \in\{0,1,2,3,4\}$, for all $j \geq 0$, either

$$
x=x_{0}+5^{1} x_{1}+5^{2} x_{2}+\ldots, \in 1+5 Z_{5} \text { or } 3+5 Z_{5},
$$

if and only if either $x_{0}=1$ or $x_{0}=3$, respectively. Let $\eta$, $\mu \in \mathbb{N}$ be the fixed subscript numbers such that $x_{\eta}=1$ and $x_{\mu}=3$. Thus, $x_{j}=0,2$ or 4 , for all $j>\eta$ and all $j>\mu$. Since, we have divided the real line into five equal line segments denoted by $0,1,2,3$, and 4 respectively. Thus, if $x_{0}=0$, then we use the function $f_{1}^{-1}$ for all $x \in N$, if $x_{0}=$ 2 , then use the function $f_{2}^{-1}$ for all $x \in N$ and if $x_{0}=4$, then use the function $f_{3}^{-1}$ for all $x \in N$. Thus, from these three cases, we obtain

$f_{1}^{-1}(x)=f_{2}^{-1}(x)=f_{3}^{-1}(x)=x_{1}+5^{1} x_{2}+\ldots,+5^{\eta-1} x_{\eta}+5^{\eta} x_{\eta+1}+\ldots$, $f_{1}^{-1}(x)=f_{2}^{-1}(x)=f_{3}^{-1}(x)=x_{1}+5^{1} x_{2}+\ldots,+5^{\mu-1} x_{\mu}+5^{\mu} x_{\mu+1}+\ldots$

again repeating the process in this manner, we obtain the general case

$$
\begin{aligned}
& f_{1}^{-1}(x)=f_{2}^{-1}(x)=f_{3}^{-1}(x)=x_{\eta}+5 x_{\eta+1}+\ldots, \\
& f_{1}^{-1}(x)=f_{2}^{-1}(x)=f_{3}^{-1}(x)=x_{\mu}+5 x_{\mu+1}+\ldots
\end{aligned}
$$

which lie in the intervals $1+5 Z_{5}$ and $3+5 Z_{5}$ respectively. Thus, we found that

$$
N \cap\left(1+5 Z_{5}\right)=\varnothing \quad \text { and } N \cap\left(3+5 Z_{5}\right)=\varnothing
$$

Hence either $x \in 1+5 Z_{5}$ or $x \in 3+5 Z_{5}$ which deduce that $x \notin N$. Hence we proved that for $x_{j} \in\{0,2,4\}, x \in N$.

Conversely, let all the variables $x=x_{0}+5^{1} x_{1}+5^{2} x_{2}+\ldots$, belong to $Z_{5}$ for all $x_{j} \in\{0,2,4\}$, and $j=0,1,2, \ldots$ Then, from Eq. (3) and (5), we can say that neither $x \in 1+5 Z_{5}$ nor $x \in 3+5 Z_{5}$ which implies that $x \notin f_{j}\left(1+5 Z_{5}\right)$ and also $x \notin f_{j}\left(3+5 Z_{5}\right)$, for $j \in W_{l}=\{1,2,3\}^{l}, l=0,1,2, \ldots .$. Thus, $x \notin\left\{\left(\bigcup_{l=0}^{\infty} \bigcup_{j \in W_{l}} f_{j}\left(1+5 Z_{5}\right)\right) \cup\left(\bigcup_{l=0}^{\infty} \bigcup_{j \in W_{l}} f_{j}\left(3+5 Z_{5}\right)\right)\right\}=Y$

Thus, $N \cup Y=Z_{5}$ and hence $x \in N$, which completes the proof of the theorem.

\section{Cantor one-fifth set as fractal string}

It is well known from the definition of fractal string that such a set consists of countably many disjoint open intervals. The lengths of which form a sequence $L=\ell_{1}, \ell_{2}$, $\ell_{3}, \ldots$, called the lengths of the string. We can assume without loss of generality that

$$
\ell_{1} \geq \ell_{2} \geq \ell_{3}, \ldots,>0
$$

where each length is counted according to its multiplicity. An ordinary fractal string can be thought of as a onedimensional drum with fractal boundary. In the literature of fractal geometry, we found a classical example of the fractal string as Cantor string. It is the set, complement of the interval $[0,1]$ of the usual ternary Cantor set. It is one of the simplest and most important example in the research of fractal string by (Lapidus and van Frankenhuijsen 2006). Information about the geometry of Cantor string like Minkowski dimension and the Minkowski measurability is obtained from its geometric zeta function. Motivated by the research of Lapidus with other researcher's (Lapidus and Hung 2008) on the Cantor string, we introduce a new Cantor one-fifth set as an example of fractal string.

The Cantor one-fifth string $\boldsymbol{N}$, is the complement of $[0,1]$ of the usual Cantor one-fifth set $F$. The Figure 3 shows the geometrical representation of Cantor one-fifth string.

Thus, we obtain

$$
\begin{aligned}
& \boldsymbol{N}=(1 / 5,2 / 5) \cup(3 / 5,4 / 5) \cup(1 / 25,2 / 25) \cup(3 / 25,4 / 25) \cup \\
& (11 / 25,12 / 25) \cup(1 / 25,2 / 25) \cup(13 / 25,14 / 25) \cup \\
& (21 / 25,22 / 25) \cup(23 / 25,24 / 25) \cup p
\end{aligned}
$$

where, $\ell_{1}=\left(l_{1}=l_{2}=1 / 5\right), \ell_{2}=\left(l_{3}=l_{4}=l_{5}=l_{6}=l_{7}=l_{8}=1 / 25\right)$ and so on. Continuing in this way, we find that the lengths

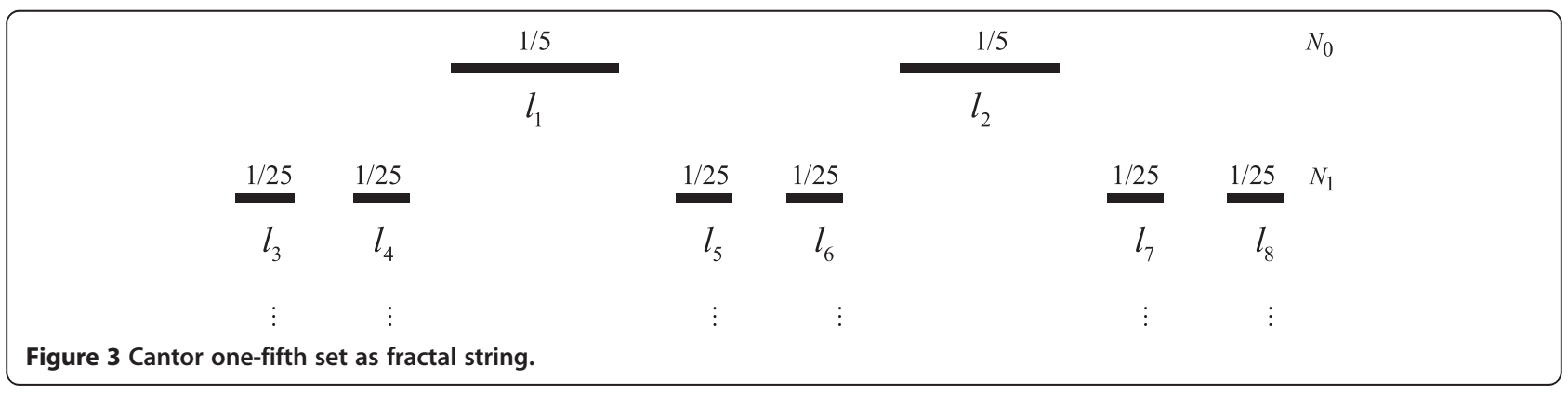




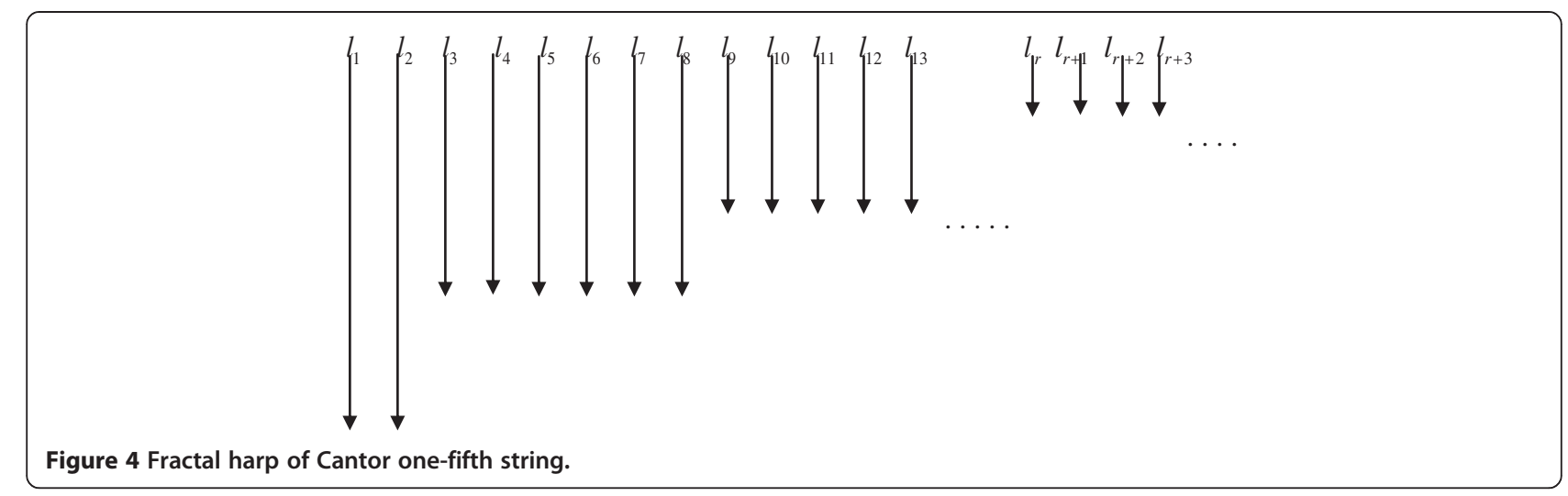

of open intervals is consist of $\ell_{k}=5^{-k-1}$ with multiplicity $m_{5^{-k-1}}=2.3^{k}$ for $k=0,1,2, \ldots$.

Thus, the geometric zeta function of the Cantor onefifth string is determined by the sequence $\boldsymbol{\aleph}$ :

$$
\begin{gathered}
\varsigma_{\boldsymbol{N}}(s)=\sum_{k=0}^{\infty} m_{k} \ell_{k}^{s}=\sum_{k=0}^{\infty} 2.3^{k} \cdot 5^{(-k-1) s}=\frac{2 \cdot 5^{s-1}}{5^{s}-3} \\
\text { for } \operatorname{Re}(s)>\log 3 / \log 5
\end{gathered}
$$

The poles of the such function are the set of complex numbers (see (Lapidus and Hung 2008), pp. 7) and given by

$$
D_{L}=\{D+i n p: n \in \mathbb{Z}\},=\{0.6826+i n 2 \pi / \log 5: n \in \mathbb{Z}\},
$$

where $D=\log 3 / \log 5=0.6826$ is the dimension of Cantor one-fifth set and $p=2 \pi / \log 5$ oscillatory period of Cantor one-fifth string $\boldsymbol{N}$, is called complex dimension of Cantor one-fifth string.

Further, representation of Cantor one-fifth string may be seen in Figure 4 using fractal harp.

\section{5-adic Cantor one-fifth set as fractal string}

Since, the construction of 5-adic Cantor one-fifth string $(\xi)$ is analogue to the usual Cantor one-fifth set. We start, by subdividing the interval $Z_{5}$ into closed subintervals

$$
\begin{aligned}
& f_{1}\left(Z_{5}\right)=0+5 Z_{5} \\
& f_{2}\left(Z_{5}\right)=2+5 Z_{5} \\
& f_{3}\left(Z_{5}\right)=4+5 Z_{5}
\end{aligned}
$$

since, fractal string is complement of the usual Cantor one-fifth set in the closed interval $[0,1]$, the remaining open subintervals after this step are given by

$$
\begin{aligned}
& Z_{5}-\bigcup_{j=1}^{2} f_{j}\left(Z_{5}\right)=1+5 Z_{5}=G_{1} \\
& Z_{5}-\bigcup_{j=2}^{3} f_{j}\left(Z_{5}\right)=3+5 Z_{5}=G_{2}
\end{aligned}
$$

then, the $G_{1} \cup G_{2}$ is the first sub-ring of self similar 5-adic Cantor one-fifth string. The lengths of $G_{1}$ and $G_{2}$ are given by using the Haar measure (Gupta and Jain 1986) as follows:

$$
l_{1}=l_{2}=\mu_{H}\left(G_{1}\right)=\mu_{H}\left(G_{2}\right)=1 / 5
$$

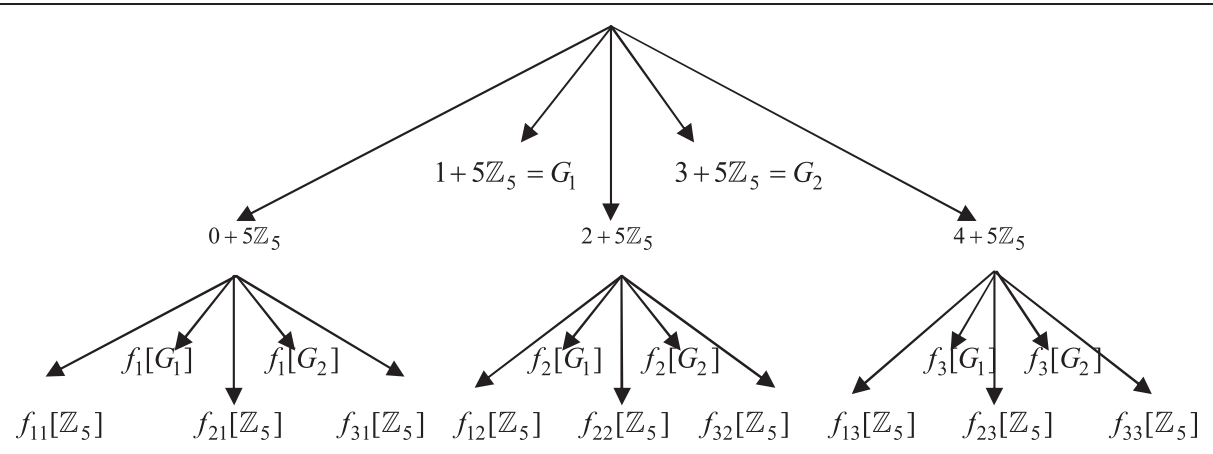

Figure 5 5-adic Cantor one fifth string via IFS. 
Again repeating the same process, by subdividing the closed intervals of first step (see Figure 1), we get

$$
\begin{array}{ll}
f_{11}\left[\mathbb{Z}_{5}\right]=0+25 \mathbb{Z}_{5}, & f_{21}\left[\mathbb{Z}_{5}\right]=10+25 \mathbb{Z}_{5}, \\
f_{31}\left[\mathbb{Z}_{5}\right]=20+25 \mathbb{Z}_{5}, & f_{12}\left[\mathbb{Z}_{5}\right]=2+25 \mathbb{Z}_{5}, \\
f_{22}\left[\mathbb{Z}_{5}\right]=12+25 \mathbb{Z}_{5}, & f_{32}\left[\mathbb{Z}_{5}\right]=22+25 \mathbb{Z}_{5}, \\
f_{13}\left[\mathbb{Z}_{5}\right]=4+25 \mathbb{Z}_{5}, & f_{23}\left[\mathbb{Z}_{5}\right]=14+25 \mathbb{Z}_{5}, \\
f_{33}\left[\mathbb{Z}_{5}\right]=24+25 \mathbb{Z}_{5} . &
\end{array}
$$

Thus, the remaining open subintervals are given by

$$
\begin{aligned}
& Z_{5}-\bigcup_{j=1}^{2} f_{j 1}\left(Z_{5}\right)=5+25 Z_{5}=G_{3}, \quad Z_{5}-\bigcup_{j=2}^{3} f_{j 1}\left(Z_{5}\right)=15+25 Z_{5}=G_{4}, \\
& Z_{5}-\bigcup_{j=1}^{2} f_{j 2}\left(Z_{5}\right)=7+25 Z_{5}=G_{5}, \quad Z_{5}-\bigcup_{j=\frac{2}{3}} f_{j 2}\left(Z_{5}\right)=17+25 Z_{5}=G_{6}, \\
& Z_{5}-\bigcup_{j=1}^{2} f_{j 3}\left(Z_{5}\right)=9+25 Z_{5}=G_{7}, \quad Z_{5}-\bigcup_{j=2}^{3} f_{j 3}\left(Z_{5}\right)=19+25 Z_{5}=G_{8} \text {. }
\end{aligned}
$$

The subring $G_{3} \cup G_{4} \cup G_{5} \cup G_{6} \cup G_{7} \cup G_{8}$ is the second set of self-similar 5-adic Cantor one-fifth string. Thus, the length is given by

$$
\begin{aligned}
l_{3} & =l_{4}=l_{5}=l_{6}=l_{7}=l_{8}=\mu_{H}\left(G_{3}\right)=\mu_{H}\left(G_{4}\right) \\
& =\mu_{H}\left(G_{5}\right)=\mu_{H}\left(G_{6}\right)=\mu_{H}\left(G_{7}\right)=\mu_{H}\left(G_{8}\right)=1 / 25 .
\end{aligned}
$$

Repeating the same process over and over again, we obtain a sequence $\ell_{1}=\ell_{2}=\ell_{3}=\ell_{4}=\ell_{5}=\ldots$... which consists of lengths $5^{-k-1}$ with multiplicity $2.3^{k}$. Using Figure 5 the 5 adic Cantor one-fifth string can also be written as follows:

$$
\begin{aligned}
& \xi=\left(1+5 \mathbb{Z}_{5}\right) \cup\left(3+5 \mathbb{Z}_{5}\right) \cup\left(5+25 \mathbb{Z}_{5}\right) \cup\left(15+25 \mathbb{Z}_{5}\right) \cup \\
& \left(7+25 \mathbb{Z}_{5}\right) \cup\left(17+25 \mathbb{Z}_{5}\right) \cup\left(9+25 \mathbb{Z}_{5}\right) \cup\left(19+25 \mathbb{Z}_{5}\right) \cup \ldots
\end{aligned}
$$

From Definition 2.3 (Lapidus and Hung 2009), the geometric zeta function of $\xi$ is given by

$$
\begin{gathered}
\varsigma_{\xi}=\left(\mu_{H}\left(1+5 Z_{5}\right)\right)^{s}+\left(\mu_{H}\left(3+5 Z_{5}\right)\right)^{s}+\left(\mu_{H}\left(5+25 Z_{5}\right)\right)^{s}+\ldots \\
=\sum_{k=1}^{\infty} m_{k} \ell_{k}^{s}=\sum_{k=1}^{\infty} 2.3^{k} \cdot 5^{(-k-1) s}=\frac{2.5^{s-1}}{5^{s}-3} \\
\text { for } \operatorname{Re}(s)>\log 3 / \log 5
\end{gathered}
$$

the poles of the such function are the set of complex numbers

$$
D_{L}=\{D+i n p: n \in \mathbb{Z}\}=\frac{\log 3}{\log 5}+i n \frac{2 \pi}{\log 5},
$$

where $D=\log 3 / \log 5=0.6826$ is the dimension of 5 -adic Cantor one-fifth string and $p=2 \pi / \log 5$ oscillatory period is the volume of the inner tubular neighborhood of $\xi$.

\section{Concluding remarks}

Based on the results, our conclusions are following:

1. In Subsection "5-adic (nonarchimedean) Cantor one-fifth set", using 5-adic integer it has been concluded that Cantor one-fifth set satisfies the nonarchimedean properties of a set and also studied that nonarchimedean Cantor one-fifth set satisfies self-similarity property using self-referential equation.

2. Further, it has been concluded that quinary Cantor one-fifth set is homeomorphic to 5-adic Cantor one-fifth set $N$ in subsection "Quinary expansion of 5 -adic Cantor one-fifth set".

3. In Subsection "Cantor one-fifth set as fractal string" and "5-adic Cantor one-fifth set as fractal string", it has been analyzed that Cantor one-fifth set and 5-adic Cantor one-fifth set both satisfy the properties of fractal string. Moreover, we found that the geometric zeta function and the complex dimension of both the sets are perfectly same.

\section{Competing interests}

The authors declare that they have no competing interests.

\section{Authors' contributions}

All authors contributed equally and significantly in writing this paper. All authors read and approved the final manuscript.

\section{Acknowledgments}

This Research is supported by the University Grant Commission of India (Grant No. 39-29/2010(SR)).

\section{Author details}

'Department of Mathematics, Maharshi Dayanand University, Rohtak 124001 Haryana, India. ${ }^{2}$ Department of Computer Science, Central University of Rajasthan, Rajasthan 305801, Rajasthan, India.

Received: 1 October 2013 Accepted: 22 November 2013 Published: 5 December 2013

\section{References}

Beardon AF (1965) On the Hausdorff dimension of general Cantor sets. Proc Camb Phil Soc 61:679-694

Cantor G (1879) Uber unendliche lineare Punktmannichfaltigkeiten, Part 1. Math Ann 15:1-7

Cantor G (1880) Uber unendliche lineare Punktmannichfaltigkeiten, Part 2. Math Ann 17:355-358

Cantor G (1882) Uber unendliche lineare Punktmannichfaltigkeiten, Part 3. Math Ann 20:113-121

Cantor G (1883a) Uber unendliche lineare Punktmannichfaltigkeiten, Part 4. Math Ann 21:51-58

Cantor G (1883b) Uber unendliche lineare Punktmannichfaltigkeiten, Part 5. Math Ann 21:545-591

Cantor G (1884) Uber unendliche lineare Punktmannichfaltigkeiten, Part 6. Math Ann 23:453-488

Chistyakov DV (1996) Fractal geometry of continuous embeddings of p-adic numbers into Euclidean spaces. Theor Math Phys 109:1495-1507

Crilly AJ, Earnshaw RA, Jones H (1991) Fractal and Chaos. Springer-Verlag, New York

Devaney RL (1992) A First Course in Chaotic Dynamical Systems. Addison Wesley Pub. Company, Inc, Holland, pp 75-79

Edgar G (2008) Measure, Topology, and Fractal Geometry. Springer Verlag, New York

Falconer K (1985) The Geometry of Fractal Sets. Cambridge University Press, Cambridge

Fleron JF (1994) A note on the history of the Cantor set and Cantor functions. Math Mag 67:136-140

Gupta VP, Jain PK (1986) Lebesgue Measure and Integration. John Wiley \& Sons, New Delhi

Gutfraind R, Sheintuch M, Avnir D (1990) Multifractal scaling analysis of diffusion-limited reactions with Devil's staircase and Cantor set catalytic structures. Chem Phys Lett 174(1):8-12 
Horiguchi T, Morita T (1984a) Devil's staircase in one dimensional mapping. Physica A 126(3):328-348

Horiguchi T, Morita T (1984b) Fractal dimension related to Devil's staircase for a family of piecewise linear mappings. Physica A 128(1-2):289-295

Hutchinson JE (1981) Fractals and self-similarity. Indiana Univ Math J 30:713-747

Koblitz N (1984) p-adic Numbers, p-adic Analysis, and Zeta-functions. Springer-Verlag, New York

Lapidus ML (1992) Spectral and fractal geometry: From the Weyl-Berry conjecture for the vibrations of fractal drums to the Riemann zeta-function. In: Bennewitz C (ed) Differential Equations and Mathematical Physics (Birmingham, 1990). Academic Press, New York, pp 151-182

Lapidus ML (2008) In Search of the Riemann Zeros: Strings, fractal membranes and noncommutative spacetimes. Amer. Math. Soc, Providence, Rl

Lapidus ML, Hung L (2008) Nonarchimedean Cantor set and string. J Fixed Point Theory Appl 3(1):181-190

Lapidus ML, Hung L (2009) Self-similar p-adic fractal strings and their complex dimensions. p-adic numbers. Ultrametric Analysis and Applications 1(2):167-180

Lapidus ML, Maier H (1995) The Riemann Hypothesis and inverse spectral problems for fractal strings. J Lond Math Soc 2(52):15-34

Lapidus ML, Pearse EPJ (2006) Tube formulas and complex dimensions of self-similar tilings. Acta Appl Math 112:91-136

Lapidus ML, Pearse EPJ (2008) Tube formulas for self-similar fractals. In: Analysis on Graphs and Its Applications. Proc Symp Pure Math, Amer Math Soc, Providence, Rl, pp 1-19

Lapidus ML, Pomerance C (1993) The Riemann zeta-function and the one-dimensional Weyl-Berry conjecture for fractal drums. Proc Lond Math Soc 3(66):41-69

Lapidus ML, van Frankenhuijsen M (2000) Fractal Geometry and Number Theory: Complex dimensions of fractal strings and zeros of zeta functions. Birkhh"auser, Boston

Lapidus ML, van Frankenhuijsen M (2006) Fractal Geometry, Complex Dimensions and Zeta Functions: Geometry and spectra of fractal strings. Springer Monographs in Mathematics, Springer-Verlag, New York

Lee JS (1998) Periodicity on Cantor sets. Comm Korean Math Soc 13(3):595-601

Hung L (2007) p-adic Fractal Strings and Their Complex Dimensions, Ph.D. Dissertation. University of California, Riverside

Madore DA (2000) A first introduction to p-adic numbers. www.madore.org/ david/ math/ padics.pdf

Mendes P (1999) Sum of Cantor sets: self-similarity and measure. Proc Amer Math Soc 127:3305-3308

Peitgen HO, Jürgens H, Saupe D (2004) Chaos and Fractals: New Frontiers of Science, 2nd edition. Springer Verlag, New York

Rani M, Prasad S (2010) Superior Cantor sets and superior Devil's staircases. Int J Artif Life Res 1(1):78-84

Robert AM (2000) A Course in p-adic Analysis, Graduate Texts in Mathematics. Springer Verlag, New York

Schikhof WH (1984) Ultrametric calculus: An introduction to p-adic analysis, Cambridge Studies in Advanced Mathematics. Cambridge Univ. Press, Cambridge

Shaver C (2010) An Exploration of the Cantor set, Mathematics Seminar. http://www. rose-hulman.edu/mathjournal/archives/2010/vol11-n1/paper1/v11n1-1pd.pdf

Vladimirov VS, Volovich IV, Zelenov El (1994) p-Adic Analysis and Mathematical Physics. World Scientific Publ, Singapore

doi:10.1186/2193-1801-2-654

Cite this article as: Kumar et al:: New 5-adic Cantor sets and fractal string. SpringerPlus 2013 2:654.

\section{Submit your manuscript to a SpringerOpen ${ }^{\circ}$ journal and benefit from:}

- Convenient online submission

- Rigorous peer review

- Immediate publication on acceptance

- Open access: articles freely available online

- High visibility within the field

- Retaining the copyright to your article

Submit your next manuscript at $\gg$ springeropen.com 